\title{
A avaliação do paciente idoso em Medicina Geral e Familiar: desafios e oportunidades
}

Maria da Conceição Balsinha,* Manuel Gonçalves-Pereira**

\section{RESUMO}

O envelhecimento da população tem consequências na sociedade em geral e na área da saúde em particular. A valorização da funcionalidade e da autonomia na velhice devem orientar a prestação de cuidados de saúde a esta população. Por outro lado, a ocultação de queixas importantes, podendo acarretar uma subidentificação de problemas de saúde, é um dos riscos na avaliação clínica destes pacientes. A avaliação estruturada de necessidades poderá contribuir para uma melhor identificação de problemas de saúde que traduzam deterioração funcional. A investigação internacional identificou cinco áreas primordiais na avaliação das necessidades de cuidados em idosos nos cuidados de saúde primários: visão e audição, mobilidade e quedas, continência de esfíncteres, memória e sofrimento psicológico. Foi sugerido que a avaliação estruturada destas áreas contribuiria com eficiência para a detecção precoce dos factores de risco que favorecem o declínio funcional e que condicionam a qualidade de vida destes pacientes. No entanto, a evidência é ainda escassa sobre a exequibilidade, na prática quotidiana, da avaliação de necessidades em Medicina Geral e Familiar.

Palavras-chave: Idoso; Funcionalidade; Avaliação de Necessidades; Medicina Geral e Familiar.

\section{INTRODUÇÃO}

A população mundial está a envelhecer, com especial destaque para a Europa. ${ }^{1}$ Portugal não é excepção nesta tendência mundial. Em 2013, Portugal foi o quinto de 27 países europeus com o índice de dependência de idosos ${ }^{*}$ mais elevado $(29,4 \%)$. ${ }^{2}$ Em 2001, o índice de envelhecimento ${ }^{\dagger}$ da população portuguesa foi de 102 e em 2011 aumentou para 128. ${ }^{3}$ Esta transformação demográfica tem motivado o debate sobre a forma como envelhecemos e sobre as implicações do envelhecimento na área da saúde e na sociedade em geral.

O centro de saúde será, provavelmente, o local mais apropriado para a prestação de cuidados médicos adequados às necessidades deste grupo etário. Em Portugal, o primeiro contacto com o Serviço Nacional de Saúde faz-se pelos Cuidados de Saúde Primários (CSP), colocando os médicos de família na linha da frente no contacto com a população. Segundo o boletim informativo da Administração Regional de Saúde de Lisboa eVale do Tejo (ARSLVT), recebido por correio electrónico em 05/07/2013, o grupo

* Médica de Família, USF Marginal, ACES Cascais.

**Departamento de Saúde Mental e CEDOC, Faculdade de Ciências Médicas, Universidade Nova de Lisboa. etário dos maiores de 64 anos foi o que registou o maior número de utilizadores no primeiro trimestre de 2013 nos centros de saúde da área desta ARS, representando 39,6\% do total. No entanto, existem constrangimentos na adequação das consultas de Medicina Geral e Familiar (MGF) à população idosa e, numa boa parte destas consultas, os cuidados prestados diferirão pouco dos prestados a um adulto de meia idade. Este artigo pretende contribuir para a reflexão sobre qual a melhor prática clínica em resposta às necessidades específicas da população idosa em CSP.

\section{A SAÚDE NO ENVELHECIMENTO}

O conceito de saúde pode ser diferente para os médicos e para os pacientes, tem mudado ao longo do tempo e depende do contexto cultural e social. ${ }^{4} \mathrm{Na}$ definição do conceito de saúde devem ser considerados os aspectos relacionados com a doença, a dolência, o sofrimento e as alterações do funcionamento físico e mental. Em 1948, a Organização Mundial de Saúde (OMS) definiu a saúde como 'um estado de completo bem estar físico, mental e social e

'População com 65 ou mais anos/população com idades compreendidas entre 15 e 64 anos.

†População com 65 ou mais anos/população com idades compreendidas entre 0 e 14 anos. 
não apenas ausência de doença ou enfermidade'. ${ }^{5}$ Esta definição vai ao encontro do modelo social de saúde (holístico) abandonando a perspectiva cartesiana que vigorava naquela época. Pretende-se ainda que a saúde deixe de ser apenas a ausência de doença, atribuindo-lhe características 'positivas' que se traduzem na presença do bem-estar.

Outra perspectiva sobre a saúde, inspirada em conceitos económicos, transforma-a num capital que reflecte a nossa herança genética. Assim, tal como o capital económico resulta do balanço entre a receita e a despesa, o capital 'saúde' depende de características genéticas, da constituição física, de factores psicológicos, do estado imunitário, entre outros. Este capital pode ser ampliado ou delapidado, ao longo da vida, dependendo das oportunidades definidas pelo contexto social. ${ }^{4}$ A metáfora apoia-se no paradigma salutogénico, cujo objectivo é identificar as causas da saúde para a poder potenciar. Deste modo, apesar de o capital 'saúde' ser inevitavelmente gasto ao longo da vida, podemos atrasar este processo garantindo reservas suficientes para ser possível envelhecer com a melhor saúde possível. O mesmo conceito está implícito na definição da promoção da saúde que, segundo a OMS, é um processo de capacitação das pessoas que lhes permite controlar e melhorar a sua saúde. ${ }^{6}$ Idealmente, este processo deve iniciar-se o mais precocemente possível e ser mantido ao longo da vida. No entanto, mesmo quando iniciado na velhice, também tem efeitos positivos sobre a saúde. ${ }^{7}$ Importa referir que promover a saúde dos indivíduos não depende apenas dos serviços de saúde, porque este processo é condicionado pelas condições sociais e económicas, pelo ambiente físico, pelo comportamento individual relativo à saúde e pelas estratégias de coping e capacitação individuais. ${ }^{8}$ As alterações biológicas do envelhecimento, assim como as redes e os papéis sociais, ${ }^{9-10}$ influenciam o estado mental, físico e social e, por isso, os componentes sócio-ambientais adquirem especial relevância e constituem vulnerabilidades para o estado de saúde na população idosa.

A manutenção da funcionalidade, entendida como a capacidade de executar autonomamente tarefas de prática corrente e necessárias a qualquer indivíduo, é inerente à promoção da saúde e a população idosa percepciona a sua saúde de acordo com a capacidade de desempenho das actividades diárias. ${ }^{11}$ Por outro lado, a incapacidade revela-se na limitação da actividade (perspectiva individual da funcionalidade) e na restrição na participação (perspectiva social da funcionalidade) dos indivíduos. Numa revisão sistemática de estudos longitudinais que avaliaram a associa- ção entre factores de risco individuais e o estado funcional de idosos na comunidade, os factores que mostraram evidência mais robusta como condicionantes da funcionalidade foram: a depressão, a limitação cognitiva, a carga de doença (burden of disease), o índice de massa corporal alterado, a limitação funcional dos membros inferiores, a escassez de contactos sociais, o baixo nível de actividade física, uma fraca auto-percepção da saúde, o tabagismo e a diminuição da acuidade visual. ${ }^{12}$ Segundo a OMS, as doenças crónicas são a principal causa de incapacidade na velhice, estimando-se que 35\% dos homens com mais de 60 anos tenham, pelo menos, duas doenças crónicas. ${ }^{13} \mathrm{O}$ impacto destas doenças deve ser medido pelos anos de vida ajustados por incapacidade, que combina o número de anos perdidos por morte prematura com o número de anos vividos com incapacidade. ${ }^{14}$ Desta forma, as doenças crónicas representam um custo significativo para os indivíduos, para as famílias, para os serviços de saúde e para a sociedade em geral. ${ }^{14}$ A valorização da funcionalidade pela população idosa e o reconhecimento da OMS quanto à importância da manutenção da autonomia e da independência na velhice, ${ }^{15}$ tem levado a um interesse crescente sobre os factores que colocam estes objectivos em risco.

\section{DETERMINANTES DE UM ENVELHECIMENTO BEM SUCEDIDO}

Num contexto de envelhecimento populacional, o debate em torno de 'acrescentar vida aos anos' ou apenas 'anos à vida' merece reflexão. Nos anos 80 do século XX, Fries ${ }^{16}$ introduziu o paradigma da compressão da morbilidade: se fosse possível adiar o aparecimento das doenças crónicas ou da incapacidade por um período de tempo superior ao do aumento da esperança de vida viver-se-iam mais anos mantendo a qualidade de vida. Este debate está relacionado com o conceito de envelhecimento bem sucedido. A OMS definiu-o como 'o processo de optimização de oportunidades para saúde, participação e segurança, no sentido de aumentar a qualidade de vida durante o envelhecimento' ${ }^{15}$ e apelidou-o de 'envelhecimento activo'. A caracterização do envelhecimento bem sucedido, quando é feito pelas pessoas idosas, considera os seguintes aspectos: saúde mental, física e social, nível de funcionalidade, satisfação com a vida, existência de sentido para a vida, segurança financeira, aprender coisas novas, realização pessoal, aparência física, produtividade, contributo na vida, sentido de humor e espiritualidade. ${ }^{17}$ Estes factores devem ser tidos em conta na planificação dos cuidados de saúde. 


\section{A ESPECIFICIDADE DOS CUIDADOS E AS PROPOSTAS DE AVALIAÇÃO}

O desenvolvimento e implementação de intervenções eficazes e eficientes para prevenir ou atrasar o aparecimento da incapacidade na velhice é uma prioridade no domínio da saúde pública. Na prestação de cuidados, da mesma forma que uma criança não é um adulto em miniatura, um idoso também não é apenas um adulto mais velho. Neste grupo etário existem vários factores que dificultam a avaliação clínica, nomeadamente a subidentificação de sintomas e dificuldades funcionais, a multipatologia, a frequência de diferentes padrões de apresentação de doença e até de patologias que são raras nas idades mais jovens. ${ }^{18}$ Esta complexidade determina que as intervenções em cuidados de saúde neste grupo etário devam ser globais, considerando as componentes biopsicossociais da saúde e alicerçadas numa avaliação multidisciplinar. ${ }^{19}$

Diversos modelos de prestação de cuidados de saúde têm sido testados em países da Europa, no Brasil, na Austrália e nos Estados Unidos da América. Alguns deles têm merecido mais atenção na literatura.

A 'avaliação geriátrica global' (comprehensive geriatric assessment) tem as suas origens nos anos 40 do século passado, resultado do trabalho da Dra. Marjory Warren..$^{20}$ Actualmente, consiste num processo diagnóstico e terapêutico, multidisciplinar, que identifica problemas biopsicossociais e funcionais em pessoas idosas com fragilidades, com o objectivo de criar um plano global para maximizar a saúde no envelhecimento. Este processo de avaliação é constituído por três etapas: 1) identificação dos pacientes elegíveis (excluídos os 'muito doentes' e os 'muito saudáveis'), 2) avaliação dos pacientes e desenvolvimento das recomendações, e 3) implementação das recomendações, dependente da adesão dos pacientes e dos clínicos. ${ }^{18}$ Esta avaliação tem sido a mais eficaz na redução do declínio funcional e na mortalidade global quando é efectuada no domicílio. No entanto, a sua realização em ambulatório tem obtido resultados contraditórios. ${ }^{21}$

A avaliação de risco (health risk appraisal) é uma outra abordagem para promover a saúde e prevenir a doença na população idosa. Consiste num questionário postal de auto-preenchimento, que engloba informação sobre o estado de saúde, a funcionalidade, os estilos de vida e hábitos, os cuidados preventivos recebidos e diversos factores psicossociais (e.g., depressão, isolamento social). As respostas obtidas são introduzidas num programa informático que permite a criação de orientações personalizadas, dirigidas ao paciente e ao médico de família. No entanto, a avaliação de risco só tem sido eficaz quando complementada com o contacto personalizado com técnicos de saúde treinados. ${ }^{22}$

O Plano Nacional para a Saúde das Pessoas Idosas ${ }^{23}$ foi criado no nosso país em 2004, com o objectivo geral de obter ganhos em anos de vida com independência, actuando sobre os determinantes da perda de autonomia e de independência. Apesar da existência deste plano nacional, não se encontra instituída nenhuma metodologia sistematizada para a avaliação gerontológica ou geriátrica das pessoas idosas em CSP.

As dificuldades mais prováveis na importação dos modelos internacionais seriam os constrangimentos económicos, a escassez de recursos humanos, a falta de formação em cuidados geriátricos e a falta de tradição relativamente ao trabalho em equipa multidisciplinar e à prestação de cuidados no domicílio. No entanto, estas dificuldades não deverão ser impeditivas de almejar a melhoria dos cuidados prestados nos CSP, com base no conhecimento existente, mas pressupondo adaptações à realidade nacional.

\section{OS DESAFIOS}

As pessoas idosas, ao contrário do que habitualmente se pensa, tendem a ocultar queixas importantes: muitas vezes associam essas queixas ao próprio envelhecimento ou têm alterações cognitivas ou depressivas que comprometem a consciência ou a verbalização das mesmas. ${ }^{18,24}$ Por outro lado, os médicos, às vezes, também interpretam algumas destas queixas como parte do processo de envelhecimento, desvalorizando ou adiando a sua resolução. ${ }^{18}$ Por isso, Freer defende que os cuidados preventivos a prestar a esta população devem ter em consideração as necessidades não reportadas. ${ }^{25}$

A eficácia do rastreio de problemas de saúde na população idosa foi muito debatida no Reino Unido na década de 80 do século passado. Nessa altura foi contratualizada, com os CSP, uma avaliação anual, no domicílio, a todos os utentes com idade superior a 74 anos, com o objectivo de identificar as necessidades não expressas e os problemas de saúde que pudessem ser alvo de intervenção para impedir a deterioração funcional..$^{26-28}$ Este modelo foi descontinuado por falta de orientações específicas relativas à avaliação dos pacientes e por deficiente avaliação dos recursos necessários, condicionando resultados heterogéneos nas diferentes unidades e falta de motivação dos mé- 
dicos de família para o processo. ${ }^{29}$ Apesar destas limitações, a avaliação do modelo permitiu saber que, do total de problemas identificados em 40 centros de saúde durante três meses, $24 \%$ (476) eram desconhecidos previamente e que $82 \%$ das pessoas com problemas identificados vieram a ser alvo de algum tipo de intervenção. ${ }^{28} \mathrm{~A}$ incontinência urinária, as limitações de mobilidade, as alterações da visão e da audição e os problemas psiquiátricos perfizeram $46 \%$ dos problemas desconhecidos. ${ }^{28}$

Faltam estudos nacionais sobre a prevalência destes problemas de saúde na população idosa. No entanto, podemos citar os resultados de um estudo exploratório em que foi calculada a sua prevalência na população com mais de 64 anos inscrita na Unidade de Saúde Familiar Marginal (USF Marginal), com base nos problemas codificados nos processos clínicos, de acordo com a International Classification of Primary Care - ICPC2. ${ }^{30}$ A prevalência da incontinência urinária foi de $5 \%$, muito inferior à encontrada na literatura (18 a 31\%). ${ }^{26,31}$ A prevalência da diminuição da audição, que pode corresponder a "presbiacusia", "surdez" ou "problema de audição" na ICPC-2, foi de $6 \%$, muito inferior aos $44 \%$ de outros estudos. ${ }^{32} \mathrm{~A}$ ICPC-2 não contém codificação específica para a ocorrência de quedas ou existência de risco de quedas, o que impossibilitou esta estimativa. Estes resultados mostram uma subcodificação em relação à prevalência esperada, mas também sugerem uma verdadeira subidentificação destes problemas de saúde nas pessoas idosas, na nossa realidade.

Apesar dos avanços recentes, a falta de formação médica pré e pós-graduada no âmbito da saúde do idoso, a ausência de avaliação de desempenho dos profissionais nesta área e o tipo de organização das consultas nos CSP dificultam a prestação de cuidados de saúde adaptados às necessidades específicas deste grupo etário. Actualmente, a avaliação de desempenho da prática clínica efectuada com a ajuda dos indicadores de contratualização engloba, por exemplo, as áreas da saúde infantil, da saúde materna e do planeamento familiar. É, no mínimo, surpreendente que não contemple o desempenho específico em 'saúde do idoso.' ${ }^{33} \mathrm{O}$ agendamento das consultas de MGF a cada quinze minutos pode levantar ainda mais dificuldades neste grupo etário. Por último, no contexto de uma cultura médica cada vez mais centrada na tecnologia, na qual se valoriza muito o "risco" e a atribuição de diagnósticos ${ }^{34}$, a dificuldade em distinguir a doença do processo normal de envelhecimento pode conduzir a práticas clínicas desvantajosas para o paciente.

\section{AS OPORTUNIDADES}

A falta de evidência sustentando a implementação generalizada de rastreios geriátricos em CSP não deve desmotivar a prática de cuidados preventivos e antecipatórios a esta população, integrados na prática do dia-a-dia. ${ }^{35} \mathrm{Os}$ médicos de família estão inseridos na comunidade, assumindo uma posição privilegiada para realizar este rastreio oportunístico pela proximidade do contacto com este grupo populacional. Na ausência de dados nacionais disponíveis sobre a taxa de utilização de consultas médicas de MGF da população de idade superior a 64 anos damos, como exemplo, a USF Marginal onde esta utilização foi de $88 \%$ nos últimos 12 meses e de $94 \%$ nos últimos 24 meses. As outras características dos médicos de família, que permitem a prestação dos cuidados de saúde multidimensionais adequados no envelhecimento, são a prestação de cuidados longitudinais, o conhecimento das famílias dos pacientes e o acesso aos seus domicílios, a gestão de todas as dimensões dos problemas de saúde e a prestação de cuidados preventivos. ${ }^{36}$

Por outro lado, o conceito de 'necessidades de cuidados' tem potencialidades enormes neste contexto. A identificação dos problemas de saúde que traduzem deterioração funcional pode ser feita mediante avaliação estruturada destas necessidades. Na área da saúde, o conceito de "necessidade" depende da possibilidade de obter benefício com a prestação de cuidados (os quais incluem medidas de educação para a saúde, medidas preventivas, de tratamento, de reabilitação ou outras). ${ }^{37}$ Uma necessidade pode considerar-se coberta (quando o problema identificado está a ser alvo de uma intervenção adequada e potencialmente benéfica) ou não coberta (se carece de intervenção adequada).$^{38} \mathrm{~A}$ principal vantagem da avaliação de necessidades é ser centrada no paciente, o que permite aprender sobre as suas necessidades e prioridades, definir estratégias para as satisfazer, utilizar os recursos existentes de forma mais efectiva e eficiente e, por último, poder influenciar políticas de saúde. ${ }^{37}$

Um instrumento para avaliação de necessidades de cuidados adaptada às pessoas idosasé a entrevista Camberwell Assessment of Need for Elderly people (CANE), ${ }^{38}$ que está traduzida e validada para Portugal. ${ }^{39-40}$ Este instrumento, inicialmente desenvolvido para ser utilizado em serviços de saúde mental, permite a triangulação das perspectivas do paciente, do cuidador (se aplicável) e do técnico de saúde responsável em 24 áreas (incluindo domínios biológicos, psicológicos e sociais, numa perspectiva multidimensio- 
nal: e.g., cuidados com a casa, actividades diárias, psicopatologia, mobilidade/quedas, continência de esfíncteres, dinheiro, contribuições sociais). Permite avaliar, perante a necessidade numa determinada área, qual o tipo (informal e formal) e o grau do apoio recebido, o nível percepcionado de ajuda necessária e sua adequação e o grau de satisfação relativa a essa ajuda. É atribuída uma pontuação relativa às necessidades existentes, cobertas e não cobertas, a qual pode servir para monitorizar avaliações futuras. Na aplicação em casos de doença crónica incapacitante podem ser usados dois itens adicionais, relativos às necessidades próprias dos cuidadores informais. ${ }^{38}$

Num estudo onde foi testada a exequibilidade da sua aplicação em CSP concluiu-se ser necessário um instrumento mais eficiente e adequado à prática quotidiana. ${ }^{41}$ Com o objectivo de construir um instrumento com essas características, Illife et al. envolveram pessoas idosas e profissionais de saúde na identificação das necessidades não cobertas mais frequentes. ${ }^{42}$ Os autores concluíram que a avaliação das necessidades nas áreas da visão e audição, da mobilidade e quedas, da continência de esfíncteres, da memória e do sofrimento psicológico correspondia a $69 \%$ das necessidades não cobertas encontradas previamente nos estudos com a aplicação do CANE. Foi assim possível seleccionar estas cinco áreas do CANE para a utilização no âmbito dos CSP com ganho de eficiência. Não obstante, é necessário replicar e eventualmente adaptar estes resultados em contextos nacionais/culturais diferentes.

Os médicos precisam conhecer e respeitar as expectativas dos seus pacientes relativamente à saúde, de modo a poderem definir uma agenda comum para a sua intervenção. O uso de entrevistas como o CANE permite uma abordagem centrada no paciente. Para além disso, a avaliação de necessidade de cuidados pode responder a um importante desafio com que lidamos nas consultas de MGF: a detecção de factores de risco para o declínio funcional. ${ }^{43} \mathrm{Te}-$ mos, neste momento, um estudo a decorrer para avaliar a exequibilidade da avaliação estruturada de necessidades, integrada na prestação de cuidados no dia-a-dia, na consulta de MGF.

\section{AGRADECIMENTOS}

Os autores agradecem à Mestre Maria João Marques pelos seus comentários às versões de trabalho do presente artigo.

\section{REFERÊNCIAS BIBLIOGRÁFICAS}

1. United Nations. World population prospects: the 2010 revision, volume I: comprehensive tables. Washington: UN; 2011 [cited 2013 May 15]. Available
from:http://esa.un.org/wpp/Documentation/pdf/WPP2010_Volume-I_Comprehensive-Tables.pdf

2. EUROSTAT. Old age dependency ration. Luxembourg: EUROSTAT; 2013 [cited 2013 Jan 8]. Available from: http://epp.eurostat.ec.europa.eu/tgm/refreshTableAction.do?tab=table\&plugin=1\&pcode=tsdde 510 \&language $=$ en

3. Instituto Nacional de Estatística. Censos 2011: resultados definitivos - Portugal. Lisboa: INE; 2012 [cited 2013 Jan 8].Available from: http://censos.ine.pt /xportal/xmain? $x$ id=CENSOS\&xpgid=ine_censos_indicador\&contexto=ind \&indOcorrCod $=0006041 \&$ selTab=tab10. Portuguese

4. Blaxter M. How is health defined? In Blaxter M, editor. Health. 2nd ed. Cambridge: Polity Press; 2010. ISBN 9780745648460

5. World Health Organization. Preamble to the Constitution of the World Health Organization as adopted by the International Health Conference, New York, 19-22 June, 1946; signed on 22 July 1946 by the representatives of 61 States (Official Records of the World Health Organization, no. 2, p. 100) and entered into force on 7 April 1948. Geneva:WHO.

6. World Health Organization. The Ottawa charter for health promotion: first international conference on health promotion. Ottawa:WHO; 1986.

7. Vaillant GE, Mukamal K. Successful aging. Am J Psychiatry. 2001;158(6): 839-47.

8. Locker D. Social determinants of health and disease. In Scambler G, editor. Sociology as applied to medicine. 6th ed. London: Saunders; 2008. ISBN 9780702029011

9. Nygren B, Aléx L, Jonsén E, Gustafson Y, Norberg A, Lundman B. Resilience, sense of coherence, purpose in life and self-transcendence in relation to perceived physical and mental health among the oldest old. Aging Ment Health. 2005;9(4):354-62.

10. Ciairano S, Rabaglietti E, Martini R, Matteo G. Older people's sense of coherence: relationships with education, former occupation and living arrangements. Ageing Soc. 2008;28(08):1075-91.

11. Bowling A. Ageing well: quality of life in old age. Berkshire: Open University Press; 2005. ISBN 9780335215096

12. Stuck AE, Walthert JM, Nikolaus T, Bula CJ, Hohmann C, Beck JC. Risk factors for functional status decline in community-living elderly people: a systematic literature review. Soc Sci Med. 1999;48(4):445-69.

13. World Health Organization. Gaining health: the European strategy for the prevention and control of noncommunicable diseases. Copenhagen: $\mathrm{WHO}$ 2006. ISBN 9289021799

14. Abegunde D, Vita-Finzi L. Preventing chronic diseases: a vital investment. Geneva:World Health Organization; 2005. ISBN 9241563001

15. World Health Organization.Active ageing: a policy framework. Geneva:WHO; 2002.

16. Fries JF. Aging, natural death, and the compression of morbidity. N Eng J Med. 1980;303(3):130-5.

17. BowlingA. Lay perceptions of successful ageing: findings from a national survey of middle aged and older adults in Britain. Eur J Ageing. 2006;3(3):12336.

18. Reuben D, Rosen S. Principles of geriatric assessment. In Halter J, Ouslander J, Tinetti M, Studenski S, High K, Asthana S, editors. Hazzard's - Geriatric medicine and gerontology. 6th ed. New York: McGraw Hill; 2009. p. 141-52. ISBN 9780071488723

19. Stuck AE, Iliffe S. Comprehensive geriatric assessment for older adults. BMJ. 2011;343:d6799.

20. Kong TK. Dr. Marjory Warren: the mother of geriatrics. J HK Geriatr Soc. 2000;10(2):102-5.

21. Ward KT, Reuben DB. Comprehensive geriatric asssessment. UpToDate [database]; 2012 [cited 2013 Jul 11].Available from: http://www.uptodate.com/ 
contents/comprehensive-geriatric-assessment?source=search_result\&search =geriatric+assessment \&selectedTitle $=1 \% 7 \mathrm{E} 46$

22. Harari D, Iliffe S, Kharicha K, Egger M, Gillmann G, von Renteln-Kruse W, et al. Promotion of health in older people: a randomized controlled trial of health risk appraisal in British general practice. Age Ageing. 2008;37(5):565-71.

23. Direção-Geral da Saúde. Programa nacional para a saúde das pessoas idosas: circular normativa n 13/DGCG, de 02/07/04. Lisboa: DGS; 2004.

24. Tulloch AJ, Moore $V$. A randomized controlled trial of geriatric screening and surveillance in general practice. J R Coll Gen Pract. 1979;29(209):733-40.

25. Freer CB. Geriatric screening: a reappraisal of preventive strategies in the care of the elderly. J R Coll Gen Pract. 1985;35(275):288-90.

26. Iliffe S, Haines A, Gallivan S, Booroff A, Goldenberg E, Morgan P. Assessement of elderly people in general practice. 2. Functional abilities and medical problems. Br J Gen Pract. 1991;41(342):13-5.

27. Iliffe S, Haines A, Gallivan S, Booroff A, Goldenberg E, Morgan P. Assessment of elderly people in general-practice. 1. Social circumstances and mental state. Br J Gen Pract. 1991;41(342):9-12.

28. Brown K, Boot D, Groom L, Williams El. Problems found in the over-75s by the annual health check. Br J Gen Pract. 1997;47(414):31-5.

29. Fletcher A. Multidimensional assessment of elderly people in the community. Br Med Bull. 1998;54(4):945-60.

30. Organização Mundial de Médicos de Família. Classificacao internacional de cuidados de saúde primários. $2^{\text {a }}$ ed. Oxford: Oxford University Press; 2011. Available from: http://www.acss.min-saude.pt/Portals/0/apmcg_ICPC \%20v\%201.7.pdf. Portuguese

31. Stoddart H, Donovan J, Whitley E, Sharp D, Harvey I. Urinary incontinence in older people in the community: a neglected problem? Br J Gen Pract. 2001;51(468):548-52.

32. Sha SH, Talaska AE, Schacht J. Age-related changes in the auditory system. In Halter J, Ouslander J, Tinetti M, Studenski S, High K, Asthana S, editors. Hazzard's - Geriatric medicine and gerontology. 6th ed. New York: McGraw Hill; 2009. p. 525-34. ISBN 9780071488723

33. Administração Central dos Sistemas de Saúde. Metodologia de contratualização: cuidados de saúde primários. Lisboa: Ministério da Saúde; 2012 [cited 2013 Jul 7]. Available from: http://www.acss.min-saude.pt/Portals/0/ 14_Mar_2012_MetodologiaContratualizaoCSP_2012.pdf. Portuguese

34. Glasziou P, Moynihan R, Richards T, Godlee F. Too much medicine: too little care. BMJ. 2013;347:f4247.
35. Freer CB. Detecting hidden needs in the elderly: screening or case finding. J R Coll Gen Pract Occas Pap. 1987;(35):26-9.

36. Allen J, Gray B, Crebolder H, Heyman J, Svab I, Ram P. The european definition of general practice / family medicine. Barcelona:WHO European Office; 2011.

37. Wright J, Williams R, Wilkinson JR. Development and importance of health needs assessment. BMJ. 1998;316(7140):1310-3.

38. Orrell M, Hancock G. The Camberwell Assessment of Need for the Elderly (CANE). London: Gaskell; 2004.

39. Gonçalves Pereira M, Fernandes L, Leuschner A, Barreto J, Falcão D, Firmino $\mathrm{H}$, et al. Versao portuguesa do CANE (Camberwell Assessment of Need for the Elderly): desenvolvimento e dados preliminares [The portuguese version of the CANE (Camberwell Assessment of Need for the Elderly): development and preliminary data]. Rev Port Saude Publica. 2007;25(1):7-18. Portuguese

40. Fernandes L, Gonçalves Pereira M,Leuschner A, Martins S, Sobral M, Azevedo LF, et al. Validation study of the Camberwell Assessment of Need for the Elderly (CANE) in Portugal. Int Psychogeriatr. 2009;21(1):94-102.

41. Walters K, Iliffe S, Tai SS, Orrell M. Assessing needs from patient, carer and professional perspectives: the Camberwell Assessment of Need for Elderly people in primary care. Age Ageing. 2000;29(6):505-10.

42. Iliffe S, Lenihan P, Orrell M, Walters K, Drennan V, Tai SS, et al. The development of a short instrument to identify common unmet needs in older people in general practice. Br J Gen Pract. 2004;54(509):914-8.

43. Eichler K, Scrabal C, Steurer J, Mann E. Preventive health risk appraisal for older people and impact on GPs' patient management: a prospective study. Fam Pract. 2007;24(6):604-9.

\section{CONFLITOS DE INTERESSE}

Os autores declaram não ter conflitos de interesses.

\section{ENDEREÇO PARA CORRESPONDÊNCIA}

Maria da Conceição Balsinha

USF Marginal - Rua Egas Moniz, 9010, $2^{\circ}$ piso.

2765-618 São João do Estoril

E-mail: mbalsinha@gmail.com

Recebido em 27-09-2013

Aceite para publicação em 20-03-2014

\section{ABSTRACT}

\section{THE ASSESSMENT OF ELDERLY PATIENTS IN GENERAL PRACTICE: CHALLENGES AND OPPORTUNITIES}

Aging of the population has implications for society in general and health systems in particular. The enhancement of function and autonomy in old age should guide the provision of health care to this group. Concealment of complaints, which may lead the doctor to miss important health problems, is one of the problems of clinical evaluation in older patients. A structured assessment of health needs may contribute to a better identification of problems linked to functional deterioration. Studies conducted in many countries have identified five key areas for assessment of the needs of the elderly in primary care: vision and hearing, mobility and falls, incontinence, memory, and emotional distress. A systematic evaluation of these areas may contribute to the early detection of risk factors that lead to functional decline, affecting the quality of life of these patients. Feasibility studies of routine needs assessment of the elderly in general practice are warranted.

Keywords: Elderly; Functionality; Needs Assessment; General Practice. 